\title{
Metric measures of interparticle interaction in an exactly solvable two-electron model atom
}

\author{
I. Nagy ${ }^{1,2}$ and I. Aldazabal ${ }^{2,3}$ \\ ${ }^{1}$ Department of Theoretical Physics, Institute of Physics, Technical University of Budapest, H-1521 Budapest, Hungary \\ ${ }^{2}$ Donostia International Physics Center DIPC, P. Manuel de Lardizabal 4, E-20018 San Sebastián, Spain \\ ${ }^{3}$ Centro de Física de Materiales (CSIC-UPV/EHU)-MPC, P. Manuel de Lardizabal 3, E-20018 San Sebastián, Spain
}

(Received 23 May 2011; published 26 September 2011)

\begin{abstract}
The exact ground-state solutions for the model of two particles in a confining harmonic oscillator potential interacting through a repulsive harmonic oscillator force are used from the standpoint of geometric distances [Phys. Rev. Lett. 106, 050401 (2011).] between wave functions and densities. The distances from the noninteracting reference state are calculated at a specified confinement by increasing the coupling of the interparticle interaction. Based on the analytic expressions for coupling-dependent geometric measures, a discussion of the Hohenberg-Kohn mapping is given.
\end{abstract}

DOI: 10.1103/PhysRevA.84.032516 PACS number(s): 31.15.ec, 03.65.-w, 03.67.-a, 71.15.Mb

\section{INTRODUCTION}

According to a recent [1] work, the metric-space viewpoint can shed light on the famous bijective mapping [2] between the ground-state (GS) wave functions and their densities, whose existence is the content of the Hohenberg-Kohn (HK) theorem. By defining geometric distances between wave functions and their corresponding densities, high-precision numerical calculations were performed [1] to explore how a change from one wave function to another affects the corresponding densities in a given system. After fixing a reference state in a nontrivial model system, some parameter of its Hamiltonian was varied in order to generate a family of ground-state wave functions and corresponding densities.

These outputs were used to calculate parametric distances (see below) to an insightful analysis on the HK mapping. Three different model systems have been used along this path of research. These are the helium isoelectronic series, a parabolically confined two-electron system, and the onedimensional Hubbard model with confined particles. It was found that the density distance $\left(D_{\rho}\right)$ not only increases monotonically as a function of the wave-function distance $\left(D_{\psi}\right)$ but also increases almost linearly, especially for He-like atoms.

Since such a remarkable linearity has apparently not been noted before in the literature on density functional theory (DFT) or on quantum mechanics, we present in this paper analytical results on $D_{\psi}(\Lambda)$ and $D_{\rho}(\Lambda)$ obtained for an exactly solvable [3-7] two-particle model atom with a tunable coupling $(\Lambda)$ to its interparticle interaction. In this model, similarly to the case of $\mathrm{He}$ isoelectronic series with Coulombic forces, one has potential energies which are homogeneous functions of the coordinates, and the motions take place in a finite region of space. Our results extend the knowledge based on numerics for mapping.

Following the lead of [1] and using the uncorrelated $(\Lambda=0$, see below) system as a reference, we define the wave-function distance and the density distance by

$D_{\psi}(\Lambda)=\left[\int_{-\infty}^{\infty} \int_{-\infty}^{\infty}\left|\psi_{0}\left(x_{1}, x_{2}\right)-\psi_{\mathrm{ex}}\left(x_{1}, x_{2}\right)\right|^{2} d x_{1} d x_{2}\right]^{1 / 2}$

$$
D_{\rho}(\Lambda)=2 \int_{0}^{\infty}\left|\rho_{0}(x)-\rho_{\mathrm{ex}}(x)\right| d x,
$$

where $\psi_{0} \equiv \psi_{\mathrm{ex}}(\Lambda=0)$ and $\rho_{0} \equiv \rho_{\mathrm{ex}}(\Lambda=0)$. The exact (ex) ground-state wave function is obtained from the Schrödinger equation with the following one-dimensional Hamiltonian:

$$
\begin{aligned}
\hat{H}= & -\frac{\hbar^{2}}{2 m}\left(\frac{d^{2}}{d x_{1}^{2}}+\frac{d^{2}}{d x_{2}^{2}}\right)+\frac{1}{2} m \omega_{0}^{2}\left(x_{1}^{2}+x_{2}^{2}\right) \\
& -\frac{1}{2} m \Lambda \omega_{0}^{2}\left(x_{1}-x_{2}\right)^{2} .
\end{aligned}
$$

Hartree atomic units, $e^{2}=\hbar=m=1$, are used from here on. The coupling, $\Lambda \in[0,0.5]$, measures the strength of a repulsive $[4,8]$ interparticle interaction. Attraction would correspond to $\Lambda<0$. Notice here that, when we apply to Eq. (1) in Sec. II approximate wave functions $\psi_{i}\left(x_{1}, x_{2}\right)$ instead of $\psi_{\mathrm{ex}}\left(x_{1}, x_{2}\right)$, a different notation $D_{\psi}^{i}(\Lambda)$ will be used for the distance from the noninteracting $(\Lambda=0)$ unique reference ground state $\psi_{0}\left(x_{1}, x_{2}\right)$.

Below we enumerate those earlier [3-8] important details for the model system which are necessary to our present study of the geometric aspects of ground-state quantum mechanics. The exact space wave function, which is normalized to unity, is given by

$$
\psi_{\mathrm{ex}}\left(x_{1}, x_{2}\right)=\left(\frac{\omega_{+} \omega_{-}}{\pi^{2}}\right)^{1 / 4} e^{-\frac{1}{2} \Omega_{1}\left(x_{1}^{2}+x_{2}^{2}\right)} e^{-\frac{1}{2} \Omega_{2} x_{1} x_{2}},
$$

where $\Omega_{1}=\left(\omega_{+}+\omega_{-}\right) / 2$ and $\Omega_{2}=\left(\omega_{+}-\omega_{-}\right)$, with $\omega_{+}=$ $\omega_{0}$ and $\omega_{-}=\omega_{0} \sqrt{1-2 \Lambda}$. The one-particle density matrix, defined from $\psi_{\mathrm{ex}}\left(x_{1}, x_{2}\right)$ as in [9], becomes

$$
\gamma_{\mathrm{ex}}\left(x_{1}, x_{2}\right)=\left(\frac{2 a-b}{\pi}\right)^{1 / 2} e^{-a\left(x_{1}^{2}+x_{2}^{2}\right)} e^{b x_{1} x_{2}} .
$$

The diagonal of $\gamma_{\mathrm{ex}}\left(x_{1}, x_{2}\right)$ gives the exact density (probability $[10,11])$ distribution,

$$
\rho_{\mathrm{ex}}(x)=\left(\frac{2 a-b}{\pi}\right)^{1 / 2} e^{-(2 a-b) x^{2}},
$$

which is needed to determine the density distance to which $\rho_{0} \equiv \rho_{\mathrm{ex}}(\Lambda=0)$. In Eqs. (5) and (6) the parameters are $b=$ $\Omega_{2}^{2} /\left(8 \Omega_{1}\right)$ and $a=\left(8 \Omega_{1}^{2}-\Omega_{2}^{2}\right) /\left(16 \Omega_{1}\right)$; thus, in terms of the 
coupling constant, $(2 a-b)=2 \omega_{0} \sqrt{1-2 \Lambda} /(1+\sqrt{1-2 \Lambda})$. Furthermore, using Eq. (3), the exact ground-state energy consists of expectation values of kinetic (first-term), confining potential (second-term), and interaction potential (last-term) energies:

$$
\begin{aligned}
E_{\mathrm{ex}}(\Lambda)= & \frac{1}{4} \omega_{0}(1+\sqrt{1-2 \Lambda})+\frac{1}{4} \omega_{0} \frac{1+\sqrt{1-2 \Lambda}}{\sqrt{1-2 \Lambda}} \\
& -\frac{1}{2} \omega_{0} \frac{\Lambda}{\sqrt{1-2 \Lambda}} .
\end{aligned}
$$

The sum of the potential energies is equal to the kinetic energy in harmony (for harmonic forces in the present case) with the virial theorem [12]. In our two-particle model system an instability occurs at the $\Lambda \rightarrow 0.5$ critical limit. For $\Lambda>0.5$ the repulsion between the particles is so strong that they cannot both remain in the external potential well [4].

One can $[5,6]$ calculate the eigenvalues $\left(P_{n}\right)$ and eigenfunctions $\left(\phi_{n}\right)$ of $\gamma_{\mathrm{ex}}\left(x_{1}, x_{2}\right)$ as well and write the spectral (s) form as follows:

$$
\gamma_{\mathrm{s}}\left(x_{1}, x_{2}\right)=\sum_{n=0}^{\infty} P_{n}(\Lambda) \phi_{n}\left(\Lambda, x_{1}\right) \phi_{n}\left(\Lambda, x_{2}\right) .
$$

As indicated, the $\Lambda$ dependence appears in two places; namely, in the eigenvalues

$$
P_{n}=\frac{2 \lambda}{1+\lambda}\left(\frac{1-\lambda}{1+\lambda}\right)^{n},
$$

where $\lambda(\Lambda)=2 \sqrt{\omega_{+} \omega_{-}} /\left(\omega_{+}+\omega_{-}\right)$, and in the eigenfunctions

$$
\phi_{n}(x)=\left(\frac{\sqrt{\bar{\omega}}}{2^{n} n ! \sqrt{\pi}}\right)^{1 / 2} H_{n}(\sqrt{\bar{\omega}} x) e^{-\frac{1}{2} \bar{\omega} x^{2}},
$$

where, with $\bar{\omega}=\sqrt{\omega_{+} \omega_{-}}=\omega_{0}(1-2 \Lambda)^{1 / 4}, \quad H_{n}(z)$ are Hermite polynomials for which $H_{n}(-x)=(-1)^{n} H_{n}(x)$. The polynomials form a complete orthonormal set. Therefore, with $\gamma_{\mathrm{s}}\left(x_{1}, x_{2}\right)$ one has $\operatorname{Tr} \gamma_{\mathrm{s}}\left(x_{1}, x_{2}\right)=1$ and $\operatorname{Tr} \gamma_{\mathrm{s}}^{2}\left(x_{1}, x_{2}\right)=\lambda(\Lambda) \leqslant$ 1 . Of course, we have the equality $\gamma_{\mathrm{s}}\left(x_{1}, x_{2}\right)=\gamma_{\mathrm{ex}}\left(x_{1}, x_{2}\right)$ between different representations. The form above for $\gamma_{\mathrm{s}}\left(x_{1}, x_{2}\right)$ allows the following interpretation: The two particles occupy independent product states $\phi_{n}\left(x_{1}\right) \phi_{n}\left(x_{2}\right)$ with $P_{n}$ probabilities. This interpretation marks the end of our survey, and now we turn to the results on the functional metrics.

\section{RESULTS}

In order to show the differences between the exact and various approximate solutions, we introduce now a parametric $\left(\Omega_{i}\right)$ product form for the wave function

$$
\psi_{i}\left(x_{1}, x_{2}\right)=\left(\frac{\Omega_{i}}{\pi}\right)^{1 / 4} e^{-\left(\Omega_{i} / 2\right) x_{1}^{2}}\left(\frac{\Omega_{i}}{\pi}\right)^{1 / 4} e^{-\left(\Omega_{i} / 2\right) x_{2}^{2}} .
$$

This form is the ground-state solution of an effective noninteracting Hamiltonian

$$
\hat{H}_{i}=-\frac{1}{2}\left(\frac{d^{2}}{d x_{1}^{2}}+\frac{d^{2}}{d x_{2}^{2}}\right)+\frac{1}{2} \Omega_{i}^{2}\left(x_{1}^{2}+x_{2}^{2}\right) .
$$

With Eq. (11) the overlap integral $O_{\mathrm{ex}, i}(\Lambda) \equiv$ $\left\langle\psi_{\mathrm{ex}}\left(x_{1}, x_{2}\right) \mid \psi_{i}\left(x_{1}, x_{2}\right)\right\rangle$ becomes

$$
O_{\mathrm{ex}, i}(\Lambda)=\frac{\left(\omega_{+} \omega_{-} \Omega_{i}^{2}\right)^{1 / 4}}{\sqrt{\frac{\Omega_{1}+\Omega_{i}}{2}+\frac{\Omega_{2}}{4}} \sqrt{\frac{\Omega_{1}+\Omega_{i}}{2}-\frac{\Omega_{2}}{4}}}
$$

after application to the prescribed double integration of the following textbook [13] result:

$$
\int_{-\infty}^{\infty} e^{-p x_{i}^{2}+2 q x_{i}} d x_{i}=\sqrt{\frac{\pi}{p}} e^{q^{2} / p} .
$$

Following the work [14] of Löwdin and Shull (LS) on natural orbitals, the variational constraint of maximum overlap results in $\Omega_{\mathrm{LS}}(\Lambda) \equiv \bar{\omega}=\omega_{0}(1-2 \Lambda)^{1 / 4}$. Then, application of $\psi_{i}\left(x_{1}, x_{2}\right)$ to calculate expectation values of the Schrödinger Hamiltonian gives

$$
E\left(\Omega_{i}\right)=\frac{1}{2}\left[\Omega_{i}+\frac{\omega_{0}^{2}}{\Omega_{i}}-\Lambda \frac{\omega_{0}^{2}}{\Omega_{i}}\right] .
$$

After variation one gets the optimal (OP) expression $\Omega_{\mathrm{OP}}(\Lambda)=\omega_{0} \sqrt{1-\Lambda}$ [6]. With $\Omega_{\mathrm{OP}}$ the virial theorem is satisfied. Imposing the constraint of the exact density $\rho_{\text {ex }}$ (i.e., not using [15] a variational principle), we get the corresponding Kohn-Sham (KS) parameter $\Omega_{\mathrm{KS}}(\Lambda)=2 \omega_{0} \sqrt{1-2 \Lambda} /(1+$ $\sqrt{1-2 \Lambda})$ to an auxiliary product function $\psi_{\mathrm{KS}}\left(x_{1}, x_{2}\right)$. Formal substitution of $\Omega_{\mathrm{LS}}(\Lambda)$ and $\Omega_{\mathrm{KS}}(\Lambda)$ in the above energetic expression would give divergent results when $\Lambda \rightarrow 0.5$ (i.e., close to the instability limit). However, the divergence is appreciably weaker with $\Omega_{\mathrm{LS}}$ than with $\Omega_{\mathrm{KS}}$. This fact heralds a superiority of optimized natural orbitals [14] to energetics. Notice that the overlap behaves, for $\Lambda \rightarrow 0.5$, as $O_{\mathrm{ex}, i}(\Lambda) \rightarrow 0$ with all these above values for $\Omega_{i}(\Lambda)$ in $\psi_{i}\left(x_{1}, x_{2}\right)$.

Now we analyze further the quality of the $\psi_{i}\left(x_{1}, x_{2}\right)$ approximate functions. Their overlap [denoted by $O_{0, i}(\Lambda)$ ] with the noninteracting $(\Lambda=0)$ reference state becomes

$$
O_{0, i}(\Lambda)=\frac{2 \sqrt{\omega_{0} \Omega_{i}}}{\omega_{0}+\Omega_{i}}
$$

which is a ratio of geometric and arithmetic means of frequencies; therefore, it is always less than or equal to unity. Notice here that $O_{0, \mathrm{LS}}(\Lambda)=\sqrt{P_{0}(\Lambda)}$. We shall use $O_{0, i}(\Lambda)$ below in the discussion of approximate wave-function distances $D_{\psi}^{i}(\Lambda)$.

When we use the effective Hamiltonian of Eq. (12) and apply to it the exact $\psi_{\mathrm{ex}}\left(x_{1}, x_{2}\right)$ correlated wave function to calculate an approximate ground-state energy, we obtain

$$
E_{i}\left(\Omega_{i}\right)=\frac{1}{4} \omega_{0}(1+\sqrt{1-2 \Lambda})+\frac{1}{4} \frac{\Omega_{i}^{2}}{\omega_{0}} \frac{(1+\sqrt{1-2 \Lambda})}{\sqrt{1-2 \Lambda}} .
$$

With the choice of $\Omega_{i}=\Omega_{\mathrm{LS}}(\Lambda)$, derived as the best [14] wave function via an overlap optimization, one gets $E_{\mathrm{LS}}\left(\Omega_{\mathrm{LS}}\right)=$ $E_{\text {ex }}(\Lambda)$. If we introduce, as in a recent work [15] on the interface of wave functions and density functionals, a variational energy difference

$$
\Delta E_{i}\left(\Omega_{i}\right) \equiv E_{i}\left(\Omega_{i}\right)-2\left(\Omega_{i} / 2\right),
$$

we get (after variation) the remarkable result of $\Omega_{i}=\Omega_{\mathrm{KS}}(\Lambda)$. In such a manner the exact density, the basic variable of the KS 
method, is recovered. On the other hand, with such an $\Omega_{\mathrm{KS}}(\Lambda)$ in Eq. (12), one still needs a certain constant [7] potential shift in order to reproduce the exact total energy using the corresponding $\left(\psi_{\mathrm{KS}}\right)$ product wave function as well.

From here on, we turn to the geometric distance defined in Eq. (1). With Eq. (4) we obtain

$$
D_{\psi}(\Lambda)=\sqrt{2}\left[1-\left(\frac{2 \sqrt{\omega_{0} \omega_{-}}}{\omega_{0}+\omega_{-}}\right)^{1 / 2}\right]^{1 / 2}
$$

This geometric measure of interparticle interaction has its maximal value at $\Lambda \rightarrow 0.5$, where $\omega_{-}(\Lambda) \rightarrow 0$. The second (overlap) term in Eq. (18) is equal to $\sqrt{\lambda(\Lambda)}$ (i.e, formally equal to the square root of the purity [6]). For a useful comparison, the distance from the noninteracting reference state with the product form of Eq. (11) is

$$
D_{\psi}^{i}(\Lambda)=\sqrt{2}\left[1-\frac{2 \sqrt{\omega_{0} \Omega_{i}}}{\omega_{0}+\Omega_{i}}\right]^{1 / 2}
$$

in terms of the above-introduced $O_{0, i}(\Lambda)$ overlap. As an illustration, the $\left(D_{\psi}\right)^{1 / 2}$ exact and the $\left(D_{\psi}^{i}\right)^{1 / 2}$ approximate distances are exhibited in Fig. 1 as a function of the coupling constant $\Lambda \in[0,0.5]$. Taylor expansions show that the limits are $D_{\psi}(\Lambda \rightarrow 0) \simeq \Lambda /(2 \sqrt{2})$ for the exact, and $D_{\psi}^{\mathrm{KS}}(\Lambda \rightarrow$ $0) \simeq \Lambda / 4$ for the approximate KS case. In the other limit of the coupling, where $\sqrt{1-2 \Lambda} \equiv \Delta$ with $\Delta \rightarrow 0$, these important geometric distances are, asymptotically, $D_{\psi}(\Lambda \rightarrow 0.5) \simeq$ $\sqrt{2}\left(1-\sqrt{2} \Delta^{1 / 4}\right)$ and $D_{\psi}^{\mathrm{KS}}(\Lambda \rightarrow 0.5) \simeq \sqrt{2}\left(1-2 \sqrt{2} \Delta^{1 / 2}\right)$. Clearly, there must be a crossing at a certain coupling. It is at around $\Lambda \simeq 0.47$.

The curve based on the variational LS approximation for the wave function is always below the exact (solid) curve. The OP curve, which is based on energy variation, is far from the exact curve at higher $\Lambda$ values [i.e., at strong $(\Lambda \rightarrow 0.5)$ interparticle repulsion]. In other words, only a part of the space entanglement is captured by this method. One can

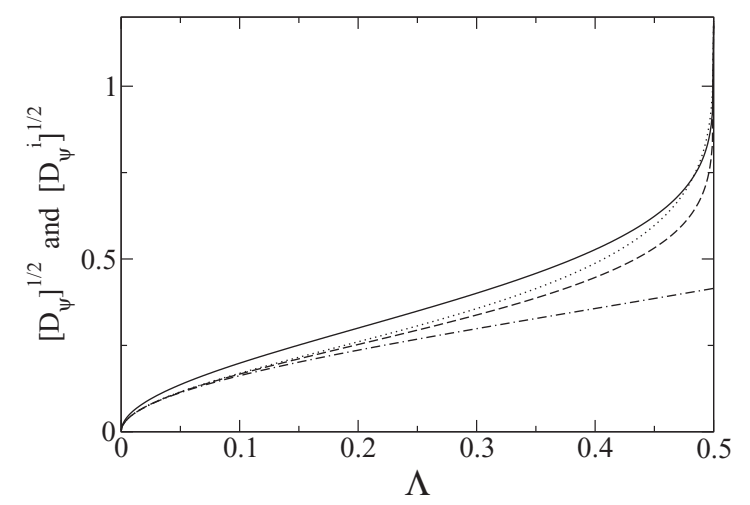

FIG. 1. Exact $\left(D_{\psi}\right)^{1 / 2}$ and the approximate $\left(D_{\psi}^{i}\right)^{1 / 2}$ dimensionless quantities as a function of the interparticle coupling constant $\Lambda \in$ $[0,0.5]$. They are calculated from Eqs. (18) and (19), respectively. The solid curve refers to the exact result. The dashed, dotted, and dash-dotted curves are based on the Löwdin-Shull $(i=\mathrm{LS})$, the Kohn-Sham $(i=\mathrm{KS})$, and the energetically optimized ( $i=\mathrm{OP})$ approximate product wave functions, respectively. All distances are calculated with respect to the noninteracting reference state. See the text for further details. easily understand this statement by considering the form of the rewritten [6] Hamiltonian

$$
\hat{H}=-\frac{1}{2}\left(\frac{d^{2}}{d x_{1}^{2}}+\frac{d^{2}}{d x_{2}^{2}}\right)+\frac{1}{2} \Omega_{\mathrm{OP}}^{2}(\Lambda)\left(x_{1}^{2}+x_{2}^{2}\right)-\Lambda \omega_{0}^{2} x_{1} x_{2} .
$$

Similarly to the conventional Hartree-Fock treatment [3] with Eq. (20), where the last term does not contribute to the groundstate energy, one gets $\Omega_{\mathrm{HF}}=\Omega_{\mathrm{OP}}$ to Eq. (11).

As mentioned above, the $D_{\psi}^{\mathrm{LS}}(\Lambda)$ function follows reasonably well (without crossing) the exact $D_{\psi}(\Lambda)$ curve. This expected characteristic prompts us to investigate a more refined approximate (a) wave function $\psi_{\mathrm{s}}^{\mathrm{a}}\left(x_{1}, x_{2}\right)$ implied by $\gamma_{\mathrm{s}}\left(x_{1}, x_{2}\right)$ in Eq. (8). We apply

$$
\psi_{\mathrm{s}}^{\mathrm{a}}\left(x_{1}, x_{2}\right)=\sum_{n=0}^{\infty}\left(P_{n}\right)^{1 / 2} \phi_{n}\left(x_{1}\right) \phi_{n}\left(x_{2}\right)
$$

to our singlet state and return to the sign problem $[14,16]$ after Eq. (22). We use this normalized $\psi_{\mathrm{s}}^{\mathrm{a}}\left(x_{1}, x_{2}\right)$ in the overlap integral performed with the reference state $\psi_{0}\left(x_{1}, x_{2}\right)$. During this calculation the following textbook [13] result is first employed before summation:

$$
\int_{-\infty}^{\infty} d x e^{-x^{2}} H_{2 n}(y x)=\sqrt{\pi} \frac{(2 n) !}{n !}\left(y^{2}-1\right)^{n} .
$$

Due to parity considerations, only these even (symmetric functions) terms $(n=0,1,2, \ldots)$ give any contribution in the infinite sum for the overlap $O_{0, \mathrm{~s}}(\Lambda)$. To find the resulting summation in the second step, we furthermore use the expression

$$
\sum_{n=0}^{\infty} \frac{(2 n) !}{2^{2 n}(n !)^{2}}\left(u^{2}\right)^{n}=\frac{1}{\left(1-u^{2}\right)^{1 / 2}}
$$

which is valid for $0<u<1$ with $u \equiv(1-\lambda) /(1+\lambda)$, to arrive at the remarkably simple form

$$
D_{\psi}^{\mathrm{s}}(\Lambda)=\sqrt{2}(1-\sqrt{\lambda})^{1 / 2},
$$

in terms of $\lambda(\Lambda)$. This result is the same as the one in Eq. (18), which is the exact $D_{\psi}(\Lambda)$. We stress, however, that, for repulsive interparticle interaction $\left[\Lambda>0\right.$; thus, $\Omega_{2}>0$ in Eq. (4)], one must use instead of the above $\psi_{\mathrm{s}}^{\mathrm{a}}$ a sign-correct Schmidt decomposition

$$
\psi_{\mathrm{s}}\left(x_{1}, x_{2}\right)=\sum_{n=0}^{\infty}(-1)^{n}\left(P_{n}\right)^{1 / 2} \phi_{n}\left(x_{1}\right) \phi_{n}\left(x_{2}\right)
$$

in order to get an $O_{\mathrm{ex}, \mathrm{s}}(\Lambda)=1$ overlap between $\psi_{\mathrm{ex}}\left(x_{1}, x_{2}\right)$ and $\psi_{\mathrm{s}}\left(x_{1}, x_{2}\right)$. On the other hand, for an attractive coupling [ $\Lambda<0$; thus, $\Omega_{2}<0$ in Eq. (4)] the $\psi_{\mathrm{s}}^{\mathrm{a}}\left(x_{1}, x_{2}\right)$ wave function results in a perfect overlap with the corresponding $\psi_{\mathrm{ex}}\left(x_{1}, x_{2}\right)$. Remarkably, the classically expected differences at $x_{1}= \pm x_{2}$ for different (in sign) couplings appear here. In the calculation of $O_{0, \mathrm{~s}}(\Lambda)$ to $D_{\psi}^{\mathrm{s}}(\Lambda)$ the sign problem does not appear due to the fact that $(-1)^{2 n}=1$.

Since only the symmetric (even) functions give contributions to Eq. (22), we might conclude that a reduced amount of information could give perfect agreement with an exact integrated result like the $D_{\psi}(\Lambda)$ function. In order to quantify 
this conclusion somewhat differently, we apply the following restricted sum of probabilities:

$$
\sum_{n=0}^{\infty} P_{2 n}(\Lambda)=\frac{1}{2}[1+\lambda(\Lambda)]
$$

obtained easily by using Eq. (9). Close to the instability limit, where $\Lambda \rightarrow 0.5$ and thus $\lambda \rightarrow 0$, the above sum tends to one half from above. Clearly, the other half is related to odd states. We note at this point that a similar importance of occupation numbers to a proper description of the molecular dissociation limit was pointed out [17] recently, based on a numerically exactly solvable one-dimensional two-center model system.

In the light of the above agreement in Eq. (24) for our wave-function-based distance, one might be tempted to conclude that not all information encoded in an exact ground-state wave function is important. We remark, however, that the interparticle interaction energy is determined $[4,9]$ by the pair function, which is $n_{\mathrm{ex}}\left(x_{1}, x_{2}\right)=\left[\psi_{\mathrm{ex}}\left(x_{1}, x_{2}\right)\right]^{2}$ in our case. Since this function is the diagonal of the two-particle density matrix, care is needed $[8,18,19]$ in attempts for a complete energetic description which rest only on the $\gamma_{\mathrm{s}}\left(x_{1}, x_{2}\right)$ matrix and its diagonal, without the exact wave function of an entangled singlet state.

The single integration in Eq. (2) for the other geometric distance is performed by dividing the integration interval $x \in[0, \infty)$ into two parts, where an intermediate $x_{0}(\Lambda)$ is determined from the condition $\rho_{0}\left(x_{0}\right)=\rho_{\mathrm{ex}}\left(x_{0}\right)$ at $\Lambda \neq 0$. In such a manner, we get

$$
x_{0} \sqrt{\omega_{0}}=\left[\frac{1}{(1-\beta)(1+\beta)} \ln (1 / \beta)\right]^{1 / 2} \text {, }
$$

where $\beta=[2 \sqrt{1-2 \Lambda} /(1+\sqrt{1-2 \Lambda})]^{1 / 2} \leqslant 1$ is a useful (see below) shorthand. Thus, using the normalization condition as well, we obtain from Eq. (2) the following:

$$
D_{\rho}(\Lambda)=4 \int_{0}^{x_{0}}\left[\rho_{0}(x)-\rho_{\mathrm{ex}}(x)\right] d x .
$$

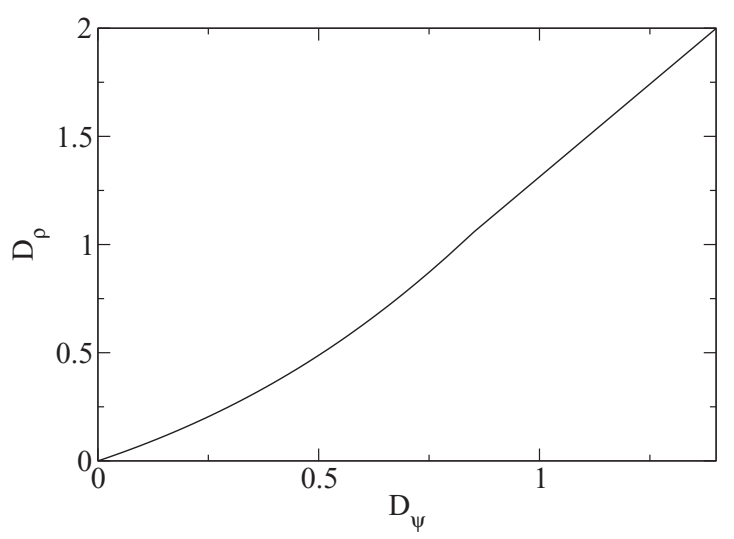

FIG. 2. Density distance $D_{\rho}$ as a function of the wave-function distance $D_{\psi}$. Both functions are based on the exact analytical solutions for the interacting Hamiltonian given by Eq. (3). See the text for further details.

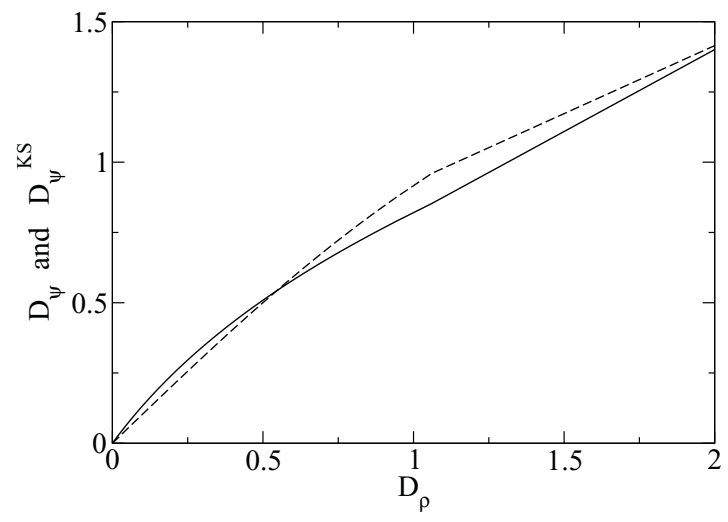

FIG. 3. Wave-function distances $D_{\psi}$ and $D_{\psi}^{\mathrm{KS}}$ as a function of the unique density distance $D_{\rho}$. The solid curve refers to the exact result and the dashed curve is based on the auxiliary Kohn-Sham product state. See the text for further details.

Since the integration results in the well-known [13] special (error) function defined by

$$
\Phi(u \sqrt{p})=2 \sqrt{\frac{p}{\pi}} \int_{0}^{u} d t e^{-p t^{2}},
$$

our closed expression for the geometric distance between densities becomes

$$
D_{\rho}(\Lambda)=2\left[\Phi\left(x_{0} \sqrt{\omega_{0}}\right)-\Phi\left(\beta x_{0} \sqrt{\omega_{0}}\right)\right] .
$$

For $\beta \rightarrow 1$, where $\Lambda \rightarrow 0$, one has $D_{\rho}(\Lambda) \sim \Lambda$, precisely as in $D_{\psi}(\Lambda \rightarrow 0)$ above. Notice here that, for our model system, one can easily calculate the approximate ( $i=\mathrm{LS}$ or $i=\mathrm{OP})$ distances $D_{\rho}^{i}(\Lambda)$ by using the $\beta_{i}=\sqrt{\Omega_{i} / \omega_{0}}$ substitutions in Eqs. (24) and (26).

In the motivating high-precision numerical study in [1] it was found that the density distance not only increases monotonically as a function of the wave-function distance, but also increases almost (especially for He-like atoms) linearly. Based on this remarkable observation, it was emphasized [1] that the bijective HK mapping seems to be a simple one. From our study, a similar qualitative statement follows by considering Fig. 2, where a related plotting method is employed. One can observe that, even at the critical limit for the interparticle coupling $(\Lambda \rightarrow 0.5)$, there is a nice linearity between distances.

Figure 3 is devoted to an illuminating comparison. Namely, the functions $D_{\psi}(\Lambda)$ and $D_{\psi}^{\mathrm{KS}}(\Lambda)$ are plotted (by solid and dashed curves, respectively) as a function of the unique $D_{\rho}(\Lambda)$. This reversed presentation is motivated by the HK mapping. The KS (dashed) curve crosses the exact curve (solid) due to its nonvariational character. On average, the dashed curve follows the path codified by the exact solution.

\section{SUMMARY AND REMARKS}

Moshinsky's two-particle model is employed in order to calculate geometrical distances between the wave functions and associated densities in an exact analytic manner. The repulsive interparticle interaction is treated via a tunable coupling $(\Lambda)$ at a specified external confinement. It is found that, for small coupling, the wave-function distance 
$D_{\psi}(\Lambda)$ and the corresponding density distance $D_{\rho}(\Lambda)$ show exactly a linear coupling dependence. Our detailed results (see Figs. 1-3) on geometric measures extend the knowledge based on recent high-precision numerical calculations performed [1] in a metric space analysis for the bijective HK mapping, which is the formal justification underlying DFT.

An essential part of the HK theorem is the fully invertible character of mapping between the ground-state density and the associated wave function. Unfortunately, the theorem only sets the stage. It does not [2] tell us how to construct the formal map $\rho \rightarrow \psi$. Our results show that, already with the simple $\psi_{\text {LS }}$ product function, one gets a $D_{\psi}^{\mathrm{LS}}(\Lambda)$ distance which deviates only moderately from the exact $D_{\psi}(\Lambda)$ even at the instability $(\Lambda \rightarrow 0.5)$ limit. On the other hand, with $\psi_{\mathrm{KS}}$ the corresponding deviation from $D_{\psi}(\Lambda)$ is not uniform [i.e., the $D_{\psi}^{\mathrm{KS}}(\Lambda)$ curve crosses the exact curve]. Further works are needed to explore more details of the formal $\rho \rightarrow \psi$ mapping. Finally, since the other part of the HK theorem refers to the invertibility of mapping between wave functions (or densities) and local potentials, it would be worthwhile to extend metric-based studies to this equally subtle [20] problem as well.

\section{ACKNOWLEDGMENTS}

Useful discussions with Professors A. K. Kazansky, N. H. March, and I. V. Tokatly are gratefully acknowledged. One of us (I.N.) thanks Professor P. M. Echenique for the warm hospitality at the DIPC. This work was supported in part by the Spanish MICINN (Project No. FIS2010-19609-C02-02).
[1] I. D’Amico, J. P. Coe, V. V. Franca, and K. Capelle, Phys. Rev. Lett. 106, 050401 (2011).

[2] R. M. Dreizler and E. K. U. Gross, Density Functional Theory (Springer Verlag, Berlin, 1990).

[3] M. Moshinsky, Am. J. Phys. 36, 52 (1968).

[4] E. R. Davidson, Reduced Density Matrices in Quantum Chemistry (Academic, New York, 1976).

[5] M. Srednicki, Phys. Rev. Lett. 71, 666 (1993).

[6] J. Pipek and I. Nagy, Phys. Rev. A 79, 052501 (2009).

[7] P. García-González, Phys. Rev. A 79, 062502 (2009).

[8] I. Nagy and J. Pipek, Phys. Rev. A 83, 034502 (2011).

[9] P.-O. Löwdin, Phys. Rev. 97, 1474 (1955).

[10] D. ter Haar, Rep. Prog. Phys. 24, 304 (1961).

[11] A. J. Coleman, Rev. Mod. Phys. 35, 668 (1963).
[12] V. B. Bobrov, S. A. Trigger, and Yu. P. Vlasov, Phys. Rev. A 83, 034501 (2011)

[13] I. S. Gradshteyn and I. M. Ryzhik, Table of Integrals, Series, and Products (Academic Press, New York, 1980).

[14] P.-O. Löwdin and H. Shull, Phys. Rev. 101, 1730 (1956).

[15] N. I. Gidopoulos, Phys. Rev. A 83, 040502 (2011).

[16] B. C. Carlson and J. M. Keller, Phys. Rev. 121, 659 (1961).

[17] N. Helbig, I. V. Tokatly, and A. Rubio, Phys. Rev. A 81, 022504 (2010).

[18] O. Gritsenko, K. Pernal, and E. J. Baerends, J. Chem. Phys. 122, 204102 (2005).

[19] K. Ebrahimi-Fard and J. M. Gracia-Bondía, J. Math. Chem. 1 (2011), doi: 10.1007/s10910-011-9822-7.

[20] N. I. Gidopoulos, Phys. Rev. B 75, 134408 (2007). 